\section{CONHECIMENTO E CONDUTA DE BOMBEIROS SOBRE TRAUMATISMO DENTOALVEOLAR}

\section{KNOWLEDGE AND CONDUCT OF FIREFIGHTERS ABOUT DENTOALVEOLAR TRAUMA}

Cristiane Alves Paz de Carvalho ${ }^{1},{ }^{*} /$ Fábio Silva de Carvalho ${ }^{1}$ / Nathally de Oliveira Rios ${ }^{1}$

\section{INTRODUÇÃO}

Traumatismo dentoalveolar caracteriza-se por uma lesão que pode envolver elementos dentários, tecidos e ossos da face. Dentre os diversos tipos de traumatismos dentários, a avulsão dental é considerada a mais frequente, sendo classificada como uma injúria que consiste no deslocamento total de um elemento dentário de seu alvéolo. Esse trauma é um dos que provocam maior apreensão aos acidentados, principalmente quando o dente envolvido é permanente. Foi observado que a prevalência desse tipo de trauma tem aumentado, por conta da prática de esportes radicais como artes marciais, dos acidentes de trânsito e da violência urbana (JETRO et al. 2013; GRANVILLE-GARCIA et al., 2007; COSTA et al., 2015).

O traumatismo dental pode levar a perdas dentais irrecuperáveis em algumas situações, durante o tratamento ou até anos depois e também no instante do acidente (ANTUNES; LEÃO; MAIA, 2012). Estudo verificou que acidentes que envolvem elementos dentários afetam, em sua maioria, a população infantil e jovem, o que pode causar um impacto negativo na qualidade de vida desses indivíduos. Além disso, essa lesão pode causar deficiência na mastigação, na fonação e também na estética, gerando um desconforto físico, psicológico e social (OLIVEIRA et al., 2013).

\section{RESUMO}

Objetivou-se analisar o conhecimento e a conduta de bombeiros militares de um município baiano com relação ao traumatismo dentoalveolar. Estudo transversal, quantitativo e descritivo, sendo utilizado um questionário semiestruturado e feita a análise estatística descritiva. Participaram 66 bombeiros, a maioria do sexo masculino $(63,6 \%)$ e com mais de 15 anos na profissão $(40,9 \%)$. Nenhum bombeiro recebeu treinamento sobre traumatismo e avulsão dentária $(100,0 \%)$ e a maioria não se sente apta a atender esse tipo de urgência $(63,3 \%)$. Nessas situações, $63,6 \%$ tentariam acalmar a vítima, conter sangramento e procurar o pedaço do dente e $72,7 \%$ encaminhariam o indivíduo para um hospital. Concluiu-se que os bombeiros desse estudo apresentam informações insuficientes sobre o tema. Evidenciou-se a necessidade de inclusão de cursos voltados para a qualificação destes profissionais, já que exercem importante papel na sociedade ao prestarem serviços de prevenção e conduta emergencial de traumas.

Palavras Chave: Avulsão Dentária. Conhecimentos. Dente. Traumatismo Dentário.

\section{ABSTRACT}

The aim of this study was to analyze the knowledge and conduct of military firefighters of a municipality in Bahia in relation to dentoalveolar trauma. Transversal, quantitative and descriptive study, using a semi-structured questionnaire and descriptive statistical analysis. Sixty-six firefighters participated, most of them male $(63.6 \%)$ and over 15 years in the profession $(40.9 \%)$. No firefighter received training on trauma and dental avulsion (100.0\%) and most did not feel able to attend this type of urgency (63.3\%). In these situations, $63.6 \%$ would try to calm the victim, contain bleeding and look for the piece of the tooth and $72.7 \%$ would refer the individual to a hospital. It was concluded that the firemen of this study present insufficient information on the subject. The need to include courses aimed at the qualification of these professionals has been evidenced, since they play an important role in society by providing preventive and emergency services for trauma.

Keywords: Tooth Avulsion. Knowledge; Tooth. Dental Trauma.

Submetido em: 05 de abr. 2019

Aceito em: 18 de ago. 2019

${ }^{1}$ Universidade Estadual do Sudoeste da Bahia, Jequié, Bahia - Brasil.

*E-mail para correspondência: capcarvalho@uesb.edu.br 
Apesar do seu caráter de urgência, os primeiros procedimentos geralmente não são executados corretamente, por conta da enorme falta de conhecimento de quem o executa. A avulsão dentoalveolar é o tipo de traumatismo dentário que exige maiores cuidados e rapidez em relação ao armazenamento do dente, porque $\mathrm{o}$ tempo extra alveolar e o meio de conservação do dente são considerados fatores determinantes para um bom prognóstico. Quando os dentes são mantidos por muito tempo fora do meio bucal, há maior ocorrência das reabsorções radiculares, responsáveis pela maioria das perdas dentárias pósreimplante (CÔRTES; BASTOS, 2004; MANGUEIRA et al., 2014; BRUNO et al. 2012).

Ao se considerar o aumento dos casos de trauma, é importante que os pais, a população, os profissionais da saúde, Serviço de Urgência e Bombeiros tenham certo conhecimento sobre os tipos de lesão para que possam agir corretamente nos primeiros socorros (SANTOS; HABECOST; GOMES et al., 2009). A atuação dos mesmos tem se mostrado de extrema importância para um prognóstico mais favorável do dente traumatizado (GRANVILLEGARCIA et al., 2007; BRUNO et al. 2012).

Segundo o Corpo de Bombeiros Militar do Tocantins (2017), na maioria dos estados brasileiros, os bombeiros são os militares que atuam junto a pessoas, animais, meio ambientes e bens materiais, e não somente no combate a incêndios, sendo seu campo de atuação bem amplo, estendendo-se às áreas de salvamento terrestre, aquático, em altura e mergulho, além do atendimento pré-hospitalar (CORPO DE BOMBEIROS MILITAR DO TOCANTINS, 2017). Contudo, não é possível afirmar que exista algum treinamento por parte dessa instituição voltado também ao atendimento a vítimas de traumatismos dentários (JETRO et al. 2013).

$\mathrm{O}$ rápido cuidado do traumatismo dentário e a saúde dental do paciente não necessitam somente de um excelente tratamento emergencial, mas dependem também do conhecimento, habilidades e decisões tomadas com eficiência e agilidade pelo cirurgiãodentista (MACENA et al., 2009). Sabe-se que a conduta correta é importante para o bom prognóstico, mas ainda há muito desconhecimento sobre $\mathrm{o}$ assunto. A falta de campanhas educativas e orientação sobre essa conduta terapêutica adequada nos casos de traumatismo e avulsão dentária podem ser fatores que diminuem a chance do sucesso clínico.

Diante desse contexto, o presente estudo teve como objetivo avaliar o conhecimento e a conduta dos bombeiros militares diante do tratamento emergencial de traumatismo dentoalveolar.

\section{METODOLOGIA}

Esta pesquisa foi desenvolvida de acordo com os princípios bioéticos preconizados pela Resolução 466/2012 do Conselho Nacional de Saúde (CNS) e foi aprovada pelo Comitê de Ética em Pesquisa da Universidade Estadual do Sudoeste da Bahia (CEP/UESB), CAAE: 59833716.0.0000.0055.

O Corpo de Bombeiros Militar da Bahia conta com Unidades Operacionais denominadas Grupamento de Bombeiros Militar (GBM), em diferentes municípios do estado, totalizando 18 Grupamentos (CORPO DE BOMBEIROS MILITAR DA BAHIA, 2019). Este estudo foi desenvolvido no $4^{\circ}$ Grupamento de Bombeiros Militar (GBM), localizado na cidade de Itabu- na, que fica a $426 \mathrm{~km}$ de distância da capital, Salvador. Caracterizou-se como um estudo transversal, de natureza quantitativa e a população de estudo foi composta pelos 91 bombeiros militares do $4^{\circ} \mathrm{GBM}$, sem distinção de sexo ou idade, que estavam no exercício de suas atividades no período de coleta dos dados da pesquisa, que ocorreu durante os meses de outubro, novembro e dezembro de 2016.

A coleta foi realizada no grupamento de trabalho dos bombeiros, onde inicialmente foi explicado o conteúdo e objetivo da pesquisa. Após concordarem em participar da pesquisa, os bombeiros assinaram o Termo de Consentimento Livre e Esclarecido (TCLE). A coleta dos dados foi feita por meio de um questionário semiestruturado composto por 12 questões objetivas, que foi elaborado com base em estudo de Jetro et al. (2013). As variáveis utilizadas para a análise foram o conhecimento e treinamento relacionado ao traumatismo dentário; a frequência e as causas mais frequentes dos casos de traumatismo dentário e a conduta diante dos casos de traumatismo e avulsão dentária.

Os dados obtidos foram tabulados em planilha do programa Office Excel 2016® e feita a análise estatística descritiva, baseada em proporções e frequências absolutas e relativas.

\section{RESULTADOS}

Dos 91 bombeiros militares do $4^{\circ} \mathrm{GBM}$, quatro se recusaram a participar e 21 não foram encontrados no grupamento nos períodos da coleta de dados. Portanto, participaram desse estudo 66 bombeiros, o que representou $72,5 \%$ da população. Dentre os 66 bombeiros participantes, $63,6 \%$ eram do sexo masculino e $36,4 \%$ do sexo feminino. Em relação à faixa etária, a 
idade média dos participantes foi de 37,1 anos.

Os bombeiros também responderam quanto tempo tinham na profissão e 40,9\% afirmaram ter mais de 15 anos. Ao serem questionados se já haviam recebido algum treinamento específico para atendimento a vítimas de traumatismo dentário, todos disseram que não (100,0\%). Como consequência, outro dado observado revelou que a maioria deles relatou não se sentir apta a atender casos de urgência em traumatismo dentário $(63,3 \%)$. Apesar de 30,3\% terem optado por mais de uma resposta quando indagados sobre o que consideravam se tratar de um traumatismo dentário, a maioria $(40,9 \%)$ acredita se tratar de uma lesão que envolve tecidos moles e ossos da face, além de elementos dentários (Tabela 1).

Tabela 1. Conhecimento e aptidão de bombeiros militares diante do traumatismo dentoalveolar, Itabuna-BA, 2016

\begin{tabular}{lrr}
\hline & N & \% \\
\hline $\begin{array}{l}\text { Conhecimento sobre traumatismo } \\
\text { dentoalveolar }\end{array}$ & & \\
Apenas dentes & 6 & 9,1 \\
Tecidos moles, ossos da face e dentes & 27 & 40,9 \\
Fratura parcial de dentes & 6 & 9,1 \\
Fratura total de dentes & 3 & 4,5 \\
Perda total de dentes após trauma & 4 & 6,1 \\
Mais de uma resposta & 20 & 30,3 \\
Treinamento específico para & & \\
traumatismo dentoalveolar & & \\
Não & 66 & 100,0 \\
Apto a atender traumatismo & & \\
dentoalveolar & & \\
Sim & 24 & 36,4 \\
Não & 42 & 63,6 \\
Total & 66 & 100,0 \\
\hline
\end{tabular}

Aproximadamente $59,0 \%$ dos bombeiros relataram que naquele grupamento há, por mês, uma frequência de zero a cinco atendimentos de casos que apresentam traumatismo dentário, sendo $48,5 \%$ dos casos relacionados aos acidentes automobilísticos. Porém, esse percentual é ainda maior já que dentre os 24 bombeiros que optaram por mais de uma causa, todos responderam acidentes automobilísticos aliados a outras causas (Tabela 2).

Tabela 2. Frequência e causas mais comuns de atendimentos relacionados a traumatismo dentoalveolar por bombeiros militares, Itabuna-BA, 2016

\begin{tabular}{lrr}
\hline & N & $\%$ \\
\hline $\begin{array}{l}\text { Frequência de atendimento de } \\
\text { traumatismo dentoalveolar }\end{array}$ & & \\
0 a 5 casos/mês & 39 & 59,1 \\
Não sei & 26 & 39,4 \\
Sem resposta & 1 & 1,5 \\
Causas mais frequentes & & \\
Acidentes automobilísticos & 32 & 48,5 \\
Não sei/Sem resposta & 9 & 13,6 \\
Violência doméstica & 1 & 1,5 \\
Mais de uma resposta & 24 & 36,4 \\
Total & 66 & 100,0 \\
\hline
\end{tabular}

Indagados sobre qual conduta adotariam ao prestarem primeiros socorros a uma vítima que sofreu traumatismo dentário, pequeno percentual disse que procuraria o pedaço do dente (1,5\%). 63,6\% dos entrevistados escolheram mais de uma resposta, e dentre elas, acalmar a vítima, conter sangramento e procurar o dente teve maior prevalência. Sobre o que fariam com o dente que saiu inteiro do lugar, 6,1\% não dariam a devida importância e rejeitariam o dente, porém, 45,5\% guardariam o elemento dentário para que a vítima entregasse ao cirurgiãodentista. Em relação à limpeza destes dentes, 40,9\% dos bombeiros afirmaram que utilizariam soro fisiológico (Tabela 3).

No que diz respeito ao item de armazenamento, 59,1\% colocariam o dente em um recipiente vazio/guardanapo. 9,1\% indicaram outras opções de armazenamento, sendo elas um recipiente com soro fisiológico, com álcool 70 (álcool etílico hidratado $70^{\circ}$ INPM - Instituto Nacional de Pesos e Medidas) ou com gelo. Sobre para onde conduziriam a vítima, $72,7 \%$ dos participantes encaminhariam para um hospital, visto que seria o lugar primário de atendimento, seguindo o protocolo aprendido no curso de formação do Corpo de Bombeiros. Porém, 18,2\% levariam a vítima a um consultório odontológico. A perda de um elemento dentário pode trazer prejuízos e 33,3\% bombeiros acreditam que a ausência do dente pode causar mastigação deficiente, problemas na fonação, prejuízos estéticos, atrapalhar o convívio social e trazer desconforto psicológico (Tabela 4).

Tabela 3. Conduta e manuseio de dentes avulsionados, por bombeiros militares, Itabuna-BA, 2016$$
\text { N } \%
$$

Primeiros socorros

Acalmar a vítima

Conter sangramento

Procurar o pedaço do dente

Levar imediatamente ao CD

Não sei

Mais de uma resposta

Conduta para avulsão dentária

Rejeitar o dente

46,1 à vítima

Tentar colocá-lo de volta na boca do $\quad 2 \quad 3,0$ indivíduo

Lavar para a vítima entregar ao

cirurgião-dentista

$\begin{array}{lll}\text { Levar imediatamente ao cirurgião- } & 5 & 7,6\end{array}$ dentista

Mais de uma resposta

Limpeza do dente

Água corrente

Soro fisiológico $\quad 27 \quad 40,9$

$\begin{array}{lrr}\text { Álcool } & 1 & 1,5\end{array}$

Não limparia $\quad 10 \quad 15,2$

$\begin{array}{lll}\text { Não sei } & 1 & 1,5\end{array}$

Mais de uma resposta $\quad 6 \quad 9,1$

$\begin{array}{lll}\text { Total } & 66 & 100,0\end{array}$ 
Tabela 4. Perspectiva de Bombeiros sobre armazenamento de dentes e prejuízos decorrentes do traumatismo dentoalveolar, Itabuna-BA, 2016

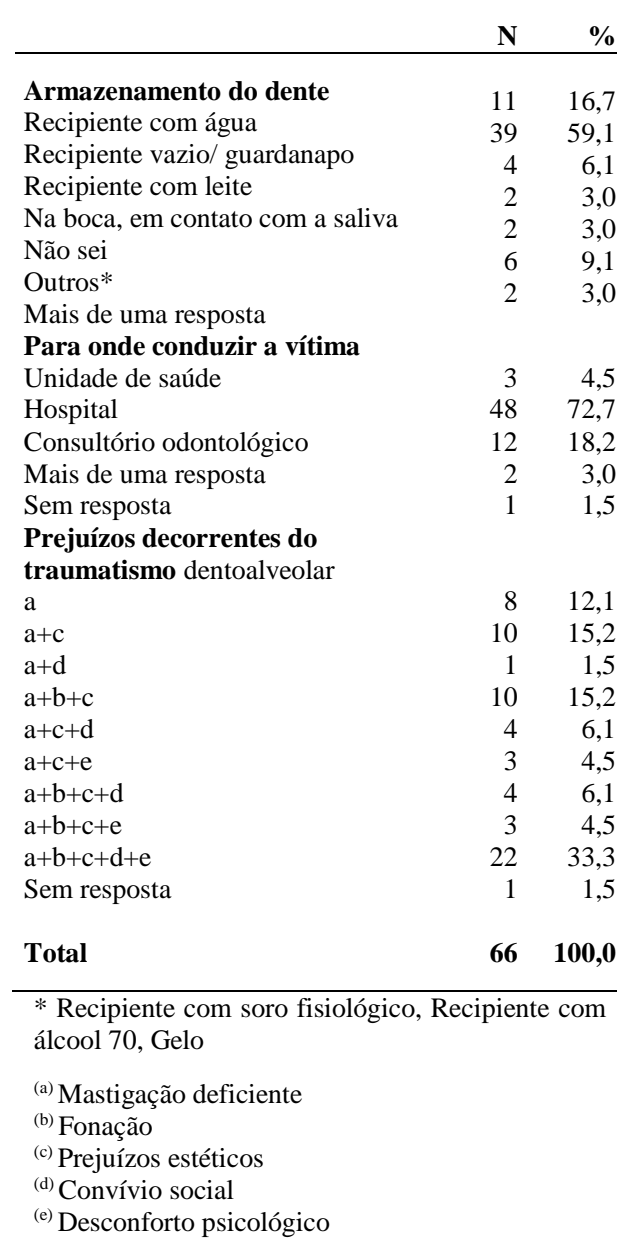

\section{DISCUSSÃO}

Traumatismos e avulsões dentárias são situações de urgências que ocorrem com frequência no consultório odontológico, sendo bastante frequentes na dentição decídua e permanente de crianças e jovens por causa de quedas, prática de esportes radicais, acidentes automobilísticos, violência urbana, lutas livres, entre outros. Muitas vezes, a população se torna passiva por falta de conhecimento ao atendimento imediato desse tipo de caso e, em contrapartida, ativa com a despreocupação com o cuidado odontológico, pois o primeiro atendimento é realizado em prontos-socorros, clínicas médi- cas ou postos de saúde (JETRO et al. 2013; SANABE et al. 2009; BITENCOURT et al. 2015; HANAN; COSTA, 2010). Em alguns países, a proporção de traumatismos dentoalveolares chega a ultrapassar a de doenças bucais persistentes, como a cárie dentária e doenças periodontais. Esses dados chamam atenção para a importância epidemiológica do traumatismo e avulsão dentária dentro da comunidade (MARCENES et al., 1999; SOUZA FILHO et al. 2011).

Protocolos para o atendimento ao paciente com traumatismo dentário têm sido descritos na literatura científica, porém a maior parte deles está direcionada para os cirurgiõesdentistas (SANTOS; HABECOST; GOMES et al., 2009; GRANVILLEGARCIA et al. 2008). Porém, muitos outros profissionais estão envolvidos em situações com esse tipo de trauma, além de pais e responsáveis, caso a vítima seja uma criança, e por isso é de extrema importância que estejam qualificados para agir corretamente mediante casos de urgência.

Os bombeiros militares estão aptos a atuar em salvamento terrestre, aquático, em altura, mergulho e atendimento pré-hospitalar (CORPO DE BOMBEIROS MILITAR DO TOCANTINS, 2017). Nesse estudo, quando indagados em como agir frente a situações de traumatismo e avulsão dentária, as respostas não foram satisfatórias. De uma forma geral, esses bombeiros demonstraram conhecimento insuficiente sobre o assunto, provavelmente pelo fato deles não terem recebido nenhum treinamento específico para atender vítimas de traumatismo dentário.

Durante a coleta de dados, foi relatado pelos participantes que a frequência de acidentes envolvendo traumatismo dentário nesse grupamen- to costuma ser de zero a cinco por mês. Talvez, um dos motivos da baixa frequência desse tipo de atendimento seja por causa da implantação, em 2004, do SAMU (Serviço de Atendimento Móvel de Urgência) no município (BRASIL, 2004). Segundo eles, tal fato reduziu em aproximadamente $80,0 \%$ a necessidade da presença da equipe dos bombeiros em determinados resgates.

Apesar de não terem informações adequadas sobre o tema, grande parte dos participantes da pesquisa afirmou que no primeiro momento tentaria acalmar a vítima, controlaria o sangramento e procuraria o dente. Caso fosse encontrado, 45,5\% responderam que ele seria lavado e guardado para que a vítima pudesse levar a um cirurgião-dentista posteriormente. Isso demonstra uma atitude conservadora, visto que o correto manejo emergencial do dente avulsionado é fundamental para o sucesso e longevidade do tratamento, pois o prognóstico está diretamente relacionado ao tempo decorrido e ao local de armazenamento, caso o dente não seja recolocado imediatamente (BITTENCOURT; PESSOA; SILVA, 2008).

$\mathrm{O}$ fator mais importante no tratamento de lesões dentárias é o tempo. Quanto maior o intervalo de tempo entre avulsão e reimplantação dentária, maior o risco de reabsorção radicular inflamatória (ZHAO; GONG, 2010). É fundamental que esses profissionais saibam como manusear o dente avulsionado, pois o ligamento periodontal presente na raiz deve ser mantido intacto. A forma correta de pegá-lo é pela coroa, sem tocar na raiz e caso seja necessário lavar para retirar alguma sujidade, deve ser feito com água corrente ou soro fisiológico, mantendo o máximo de estrutura periodontal possível (GRANVILLE- 
GARCIA et al., 2007; COSTA et al., 2015; POI et al. 2013). Com relação ao armazenamento, o leite fresco frio é superior à saliva na manutenção da vitalidade do elemento dentário, tanto in vitro como in vivo, aumentando as chances de sucesso no reimplante (ZHAO; GONG, 2010). Sendo assim, um recipiente com esse líquido seria a maneira mais indicada para armazenar o dente avulsionado. Entretanto, como visto nesse estudo, a maior parte dos participantes acomodaria o dente em um recipiente vazio/guardanapo, o que é desfavorável, por comprometer a vitalidade pulpar e a viabilidade do tecido conjuntivo de aderir novamente (GRANVILLE-GARCIA et al., 2007).

Apesar de saberem da necessidade de conduzir a vítima com rapidez a uma unidade de saúde ou consultório odontológico e deixar o dente o mínimo possível fora do alvéolo, os bombeiros afirmaram que, na maioria dos casos, encaminhariam a vítima para o hospital mais próximo, sendo pouco mencionado o atendimento por um cirurgião-dentista como opção de tratamento. Isso pode ser devido ao fato de que durante o curso de formação, são orientados a encaminhar a vítima ao local mais próximo em que possam ter atendimento médico de urgência, ou seja, o hospital. Vale lembrar que quando é necessária a presença de bombeiros em uma cena de acidente, geralmente se tratam de acidentes graves, em que o indivíduo corre perigo de morte (JETRO et al. 2013; CORPO DE BOMBEIROS MILITAR DO TOCANTINS, 2017).

O traumatismo dentário pode afetar drasticamente a qualidade de vida de uma pessoa. Além de dor e desconforto, a perda de um elemento dentário causa prejuízos estéticos, acarretando problemas psicossociais, dificuldade na mastigação e fonação, entre outros (OLIVEIRA et al., 2013). Apesar de 33,3\% dos bombeiros desse estudo terem relatado todos os prejuízos mencionados acima, ressalta-se que percentual bem próximo $(30,4 \%)$ relatou que o traumatismo dentário pode causar prejuízos apenas para mastigação, fonação e estética, não demonstrando a percepção de outras dimensões, como o desconforto psicológico e o convívio social.

\section{CONCLUSÃO}

De acordo com resultados obtidos, pode-se concluir que os bombeiros militares desse estudo possuem um conhecimento limitado sobre traumatismo dentoalveolar. A grande maioria não sabe como proceder nessas situações e desconhece a importância do correto manuseio e armazenamento do elemento dentário.

Dessa forma, evidenciou-se a necessidade de inclusão de informações sobre o tema durante o curso de formação desses bombeiros e também por meio de palestras, visando à qualificação destes profissionais, que exercem um importante papel na sociedade ao prestarem serviços de prevenção e conduta emergencial de traumas.

Deve-se destacar que esse estudo apresenta limitações, por ter sido realizado com uma amostra de bombeiros militares de um município baiano. Ressalta-se que as inferências obtidas podem não representar os bombeiros militares de um modo geral. Entretanto, verificou-se que se faz necessária e importante a realização de novos estudos que tragam informações sobre o conhecimento e a conduta adotada por estes profissionais frente ao tratamento emergencial de vítimas com traumatismo dentoalveolar, para que estratégias possam ser implementadas para melhor habilitá-los para o atendimento nessas situações.

\section{REFERÊNCIAS}

ANTUNES, L.A.A.; LEÃO, A.T.; MAIA, L.C. Impacto do traumatismo dentário na qualidade de vida de crianças e adolescentes: revisão crítica e instrumentos de medida. Ciênc. saúde coletiva., v.17, n.12, p.3417-3424.

2012. Disponível em:

$<$ http://www.scielo.br/scielo.php?pid= S1413-

232012001200026\&script=sci_abstrac $\mathrm{t} \&$ tlng $=\mathrm{pt}>$

BITENCOURT, S.B.; CUNHA, A.I.O.; OLIVEIRA, D.W.R. et al. Abordagem terapêutica das fraturas dentárias decorrentes do traumatismo dentário. Rev Odontol Araçatuba., v.36, n.1, p.24-29. 2015. Disponível em: <

http://apcdaracatuba.com.br/revista/20 15/07/03.pdf>

\section{BITTENCOURT, A.M.; PESSOA,} O.F.; SILVA, J.M. Avaliação do conhecimento de professores em relação ao manejo da avulsão dentária em crianças. Rev Odontol UNESP., v.37, n.1, p.15-19. 2008. Disponível em: < http://s3.amazonaws.com/host-articleas-

sets/rou/588018407f8c9d0a098b4b34/f ulltext.pdf>

\section{BRASIL. MINISTÉRIO DA SAÚDE.}

Portaria $\mathrm{n}^{\circ}$ 2.216/GM/MS, de 14 de outubro de 2004, que habilita Serviço de Atendimento Móvel de Urgência (SAMU 192) nos Municípios de Eunápolis (BA), Itabuna (BA) e Jequié (BA). Brasília, 2004. Disponível em: <http://bvsms.saude.gov.br/bvs/saudel egis/gm/2004/prt2216_14_10_2004.ht ml>. Acesso em 04 abr 2019. 
BRUNO, K.F.; SOUZA, B.L.; OLIVEIRA, D.A. et al. Conhecimento de profissionais de Educação Física frente ao tratamento emergencial de dentes permanentes avulsionados. Rev Odontol UNESP., v.41, n.4, p.267-272.

2012. Disponível em: <

http://s3.amazonaws.com/host-articleas-

sets/rou/5880192d7f8c9d0a098b500a/f ulltext.pdf>

CORPO DE BOMBEIROS MILITAR DA BAHIA. Bahia, 2019. Disponível em: <

http://www.cbm.ba.gov.br/unidadesoperacionai>. Acesso em 11 out. 2019.

\section{CORPO DE BOMBEIROS MILITAR} DO TOCANTINS. Tocantins; 2017.

Disponível em:

$<$ http://bombeiros.to.gov.br/quemsomos/reas-de-atuacao/>. Acesso em 26 abr. 2017.

CÔRTES, M.I.S.; BASTOS, J.V. Lesões traumáticas na dentição permanente. In: ESTRELA, C. Ciência endodôntica. São Paulo: Artes Médicas. 2004, p.799-918.

COSTA, H.S.; LIMA, M.C.P.S.; LEITE, K.V.M. et al. Conhecimento de acadêmicos do curso de educação física sobre avulsão dentária e uso de protetor bucal. Rev Odontol Araçatuba., v.36, n.2, p.36-40. 2015. Disponível em: <

http://apcdaracatuba.com.br/revista/20 15/12/TRABALHO\%206.pdf>

GRANVILLE-GARCIA, A. F.; LIMA, E.M.; SANTOS, P.G. et al. Avaliação do Conhecimento dos Professores de Educação Física de Caruaru-PE Sobre Avulsão Reimplante. Pesq Bras Odontopediatria Clin Integr. João Pessoa, v.7, n.1, p.15-20. 2007. Disponível em:
$<$ http://revista.uepb.edu.br/index.php/p boci/article/viewFile/203/150>

GRANVILLE-GARCIA, A.F.; MENEZES, V.A.; LOPES, I. et al. Conduta terapêutica dos cirurgiões-dentistas em relação aos traumatismos dentários. Arq Ciênc Saúde Unipar., Umuarama, v.12, n.3, p.239-247. 2008. Disponível em: < http://revistas.unipar.br/index.php/saud e/article/view/2541/1984>

HANAN, S.A.; COSTA, S.K. Conhecimento dos professores de $1^{\mathrm{a}}$ a $4^{\mathrm{a}}$ série de escolas públicas municipais de Manaus/AM frente a avulsão dentária.

\section{Pesqui Bras Odontopediatria Clin}

Integr., João Pessoa, v.10, n.1, p.27-

33. 2010. Disponível em: <

http://revista.uepb.edu.br/index.php/pb oci/article/viewFile/806/417>

JETRO, V.; MORAIS, H.H.A.; DIAS, T.G.S. et al. Traumatismo dentoalveolar: nível de conhecimento e conduta de urgência de bombeiros do município de Caicó-RN. Rev. Cir. Traumatol. Buco-Maxilo-Fac., Camaragibe, v.13, n.2, p.101-108. 2013. Disponível em:

<https://www.researchgate.net/publicat ion/284454156_Traumatismo_dentoal veo-

lar_nivel_de_conhecimento_e_conduta _de_urgencia_dos_bombeiros_do_mu nicipio_de_Caico-RN>

MACENA, M.C.B.; LEITE, A.C.; COLARES, V. et al. Protocolo clínico de avaliação e conduta no traumatismo dentário. RBPS., v.22, n.2, p.120-127. 2009. Disponível em: <

https://www.researchgate.net/publicati on/279677915_Protocolo_clinico_de_ avalia-

cao_e_conduta_no_traumatismo_denta rio_-_doi105020180612302009p120>
MANGUEIRA, L.L; MORAES, T.M.; ROSENDO, R.A. et al. Avaliação do nível de conhecimento dos estudantes de Educação Física sobre avulsão dentária. Rev. bras. odontol., Rio de Janeiro, v.71, n.2, p.183-187. 2014. Disponível em: <

http://revista.aborj.org.br/index.php/rb o/article/view/548/434>

MARCENES, W.; al BEIRUTI, N.; TAYFOUR, D. et al. Epidemiology of traumatic injuries to the permanent incisor of 9-12 years old school children in Damascus, Syria. Endod Dent Traumatol., v.15, n.3, p.117-123. 1999. Disponível em: < https://www.ncbi.nlm.nih.gov/pubmed /10530154>

OLIVEIRA, M.J.L.; DIAS, V.O.; SANTOS, K.K.F. et al. Análise do Conhecimento dos Pais/Responsáveis pelas Crianças Atendidas na Clínica Infantil da Unimontes sobre Traumatismos Dentários. Pesq Bras Odontopediatria Clin Integr., João Pessoa, v.13, n.2, p.189-196. 2013. Disponível em: <

http://revista.uepb.edu.br/index.php/pb oci/article/viewFile/1621/1060>

POI, W.R.; SONODA, C.K.; MARTINS, C.M. et al. Storage media for avulsed teeth: a literature review. Braz Dent J., Ribeirão Preto, v.24, n.5, p.437-445. 2013. Disponível em: < http://www.scielo.br/scielo.php?script $=$ sci_arttext\&pid=S010364402013000500437

\section{SANABE, M.E.; CAVALCANTE,} L.B.; COLDEBELLA, C.R. et al. Urgências em traumatismos dentários: classificação, características e procedimentos. Rev Paul Pediatr., v.27, n.4, p.447-451. 2009. Disponível em: $<$ 
http://www.scielo.br/pdf/rpp/v27n4/v2

7n4a15.pdf>

SANTOS, M.E.; HABECOST, A.P.;

GOMES, F.V. et al. Parent and caretaker knowledger about avulsion of permanent teeth. Dent Traumatol., v.25, n.2, p.203-208. 2009 Disponível em: <

https://www.ncbi.nlm.nih.gov/pubmed /19290901>

SOUZA FILHO, M.D.; MOURA, M.S.; ARAÚJO, R.S.R.M. et al. Prevalência de traumatismo dentário em pré-escolares de Teresina, PI. Arq

Odontol., Belo Horizonte, v.47, n.1, p.18-24. 2011. Disponível em: < http://revodonto.bvsalud.org/pdf/aodo/ v47n1/a03v47n1.pdf>

ZHAO, Y. GONG, Y. Knowledge of emergency management of avulsed teeth: a survey of dentists in Beijing, China. Dental Traumatology., v.26, p. 281-284. 2010. Disponível em: < https://www.ncbi.nlm.nih.gov/pubmed /20572844> 